\title{
Porous ceramic scaffolds with complex architectures
}

E. Munch, J. Franco, S. Deville ${ }^{1}$, P. Hunger, E. Saiz and A. P. Tomsia

Materials Sciences Dvision, Lawrence Berkeley National Laboratory, Berkeley CA 94720

\begin{abstract}
This work compares two novel techniques for the fabrication of ceramic scaffolds for bone tissue engineering with complex porosity: robocasting and freeze casting. Both techniques are based on the preparation of concentrated ceramic suspensions with suitable properties for the process. In robocasting, the computer-guided deposition of the suspensions is used to build porous materials with designed three dimensional (3-D) geometries and microstructures. Freeze casting uses ice crystals as a template to form porous lamellar ceramic materials. Preliminary results on the compressive strengths of the materials are also reported.
\end{abstract}

\section{Introduction}

Most strategies to regenerate bone depend on 3-D porous structures (scaffolds) to support cell attachment, proliferation, and differentiation. Perhaps one of the most critical challenges in bone tissue engineering is to develop custom-designed scaffolds, tailored to mimic natural biological templates and with mechanical properties matching those of the surrounding tissue. The ideal scaffold for bone regeneration should be formed using an osteoconductive or osteinductive porous material that will form a secure bond with the tissues by allowing and encouraging invasion of new cells. The porosity must be interconnected to allow the ingrowth of cells, vascularization, and diffusion of nutrients. The scaffold must provide the necessary support for cells to proliferate and slowly degrade and resorb as the tissue structures grow in vitro and/or in vivo [1]. In conventional porous scaffold fabrication methods [2-10] it is difficult to precisely control pore size, geometry and spatial distribution, to create internal channels within the scaffold for vascularization, and to produce scaffolds with arbitrary and complex 3-D anatomical shapes.

In this work we describe two new techniques for the fabrication of scaffolds with complex pore distributions: robotic assisted deposition and freeze casting. Both techniques are based on the preparation of water-based ceramic suspensions. Robotic assisted deposition (robocasting) allows the fabrication of polymer and ceramic scaffolds without the need for a sacrificial support material or mold [12-14]. This technique consists of the robotic deposition of inks capable of fully supporting their own weight during assembly, thanks to their carefully tailored composition and viscoelastic properties. In freeze casting, porous lamellar ceramic or polymeric materials are built through the controlled directional freezing of concentrated suspensions [15-19]. Here we will show how these techniques can be used here to prepare porous hydroxyapatite (HA) ceramics. Hydroxyapatite is an osteoconductive ceramic closely related to the inorganic component of bone and one of the leading candidates for the fabrication of porous inorganic scaffolds for bone tissue engineering. However, both technologies can be easily extended to the fabrication of scaffolds using different polymer and ceramic materials (e.g., other calcium phosphates such as tri-calcium phosphate or bioactive glasses).

\footnotetext{
${ }^{1}$ Current address: Laboratoire de Synthèse et Fonctionnalisation des Céramiques FRE2770 CNRS/St Gobain CREE

550, Avenue Alphonse Jauffret, BP 224

84306 Cavaillon CEDEX
} 


\section{Experimental}

Robocasting inks were prepared by the following two different approaches. The first of them starts with the preparation of a stable hydroxyapatite suspension with $40-45 \mathrm{vol} \%$ of powder in distilled water. The stability of the suspension was achieved by dissolving the appropriate amount of Darvan ${ }^{\circledR} \mathrm{CN}$ dispersant (R.T. Vanderbilt, Norwalk, CT) in water and then gradually adding the hydroxyapatite powder (Hydroxyapatite\#30, Trans-Tech, Adamstown, MD) while shaking vigorously after each addition to improve its homogeneity and stability using a shaker and zirconia balls. An appropriate amount ( $7 \mathrm{mg}$ per $\mathrm{ml}$ of solution) of previously dissolved hydroxypropyl methylcellulose (Methocel F4M, Dow Chemical Company, Midland, MI) was then added to the mixture to increase viscosity. Subsequently, the ink was gellified by adding the proper amount of polyethylenimine (PEI) as flocculant. The viscosity of the final ink was modified to desired consistency by adjusting its $\mathrm{pH}$ with $\mathrm{HNO}_{3}$ or $\mathrm{NH}_{4} \mathrm{OH}$ as needed. Each addition to the mixture was followed by mixing for about 1 hour in the shaker.

The second approach is based on the use of a commercial hydrogel, Pluronic ${ }^{\circledR}$ F-127, (BASF corp), as a printing vehicle. Pluronic consists of approximately $70 \mathrm{wt} \%$ ethylene oxide and $30 \mathrm{wt} \%$ propylene oxide. It is soluble in cold water $\left(\mathrm{T} \sim 5^{\circ} \mathrm{C}\right)$ and it forms a hydrogel at room temperature. The HA powders are dispersed in a mixture of water and pluronic (10-30 wt $\%$ of Pluronic) with the appropriate amount of Darvan ${ }^{\circledR} \mathrm{CN}$ as a dispersant. After adding the HA, the suspension is placed for 1 hour in the shaker using zirconia balls as mixing media in order to achieve a homogeneous dispersion and break large aggregates.

In both cases, the final quality of the inks was assessed in terms of printability, measured as the minimum tip diameter suitable to extrude the ink without clogging, and stability (i.e., shape retention capacity during drying and sintering) of the assembled structures.

In order to formulate stable hydroxyapatite slurries with the right composition for the freeze drying process, the sedimentation of slurries on narrow glass test tubes $(\sim 1 \mathrm{~cm}$ in diameter) was analyzed as a function of the dispersant (typically ammonium polymethacrylate anionic dispersant Darvan C or Darvan 811, R. T. Vanderbilt Co., Norwalk, CT) and binder (PVA) concentrations. Once the optimum compositions were identified, the hydroxyapatite slurries were prepared by mixing distilled water with the dispersant, the binder and the hydroxyapatite powder in various contents ranging from 15 to 40 vol\%. Slurries were ball-milled for 20 hours with alumina balls and de-aired by stirring in a vacuum dessicator. Controlled unidirectional freezing of the slurries was done by pouring them into a Teflon mold, with two copper rods on each side which were cooled using liquid nitrogen. Freezing kinetics were controlled by heaters placed on the metallic rods and thermocouples placed on each side of the mold [15-17]. Frozen samples were freeze dried for $24 \mathrm{hrs}$ in order to sublimate the water and leave the green ceramic scaffold.

Sintering of the green bodies produced using both techniques was done in an air furnace with dwell temperatures ranging between $1250^{\circ} \mathrm{C}$ to $1350^{\circ} \mathrm{C}$ (heating rate $3^{\circ} \mathrm{C} / \mathrm{min}$ ) and sintering times from 2 to 6 hours. In the case of materials prepared by robocasting, the samples were dried in air at room temperature for 24 hours and then at $400^{\circ} \mathrm{C}\left(1^{\circ} \mathrm{C} / \mathrm{min}\right.$ heating rate) for 1 hour to evaporate organics prior to sintering. 
The starting powders and sintered samples were characterized by X-ray diffraction and scanning electron microscopy (SEM). In addition, the sintered density and open porosity of the samples was characterized using Archimede's method. Preliminary uniaxial compression tests were performed using crosshead speeds ranging between 0.01 to $0.015 \mathrm{~mm} / \mathrm{s}$ in $\sim 4 \times 4 \times 5 \mathrm{~mm}^{3}$ samples cut from the scaffolds using a diamond saw.

\section{Results and discussion}

\section{Robocasting}

Colloidal inks developed for robocasting must satisfy two important criteria [13]: first, their viscoelastic properties must allow them to flow through a deposition nozzle and then "set" immediately so that shape is retained as additional layers are deposited, or when they span gaps in the underlying structure (Figs. 1 and 2). Second, the suspensions must have a high solid volume concentration to minimize shrinkage during drying so that the particle network is able to resist the involved capillary stresses. The stability of these high-solids-loading suspensions requires high dispersive forces between particles, and therefore the role of dispersant is critical. The amount of dispersant has to be adjusted to efficiently coat the particles in the suspension, but an excess will cause flocculation of the particles due to the depletion effect [13]. It was possible to determine that for the average ceramic particle size used in this work $(\sim 1-2 \mu \mathrm{m})$ the optimal concentration of dispersant is around $0.5 \%$ relative to powder weight.

Robocasting requires a pseudoplastic suspension with a yield stress high enough to prevent shape changes on the printed structure under its own weight. Therefore, the welldispersed high-solids-loading suspensions are not yet functional for robocasting. Their rheology must be altered to form partially flocculated suspensions where a loosely bound particle network is formed [13]. In the first approach PEI was used as flocculant to create the desired links between particles through interaction between its positively charged amine groups and the negatively charged carboxylic acid groups of the dispersant covering the HA particles. The optimal amount of PEI was estimated to be around $0.4 \mathrm{wt} \%$ relative to water content. The final tuning of the viscoelastic properties of the suspension is achieved by slightly adjusting its $\mathrm{pH}$ with $\mathrm{HNO}_{3}$ or $\mathrm{NH}_{4} \mathrm{OH}$ as needed. The $\mathrm{pH}$ of the suspension controls the activity of both dispersant and flocculant and, therefore, the strength of the bonds between the particles and its ability to form the desired network. In this system the optimum $\mathrm{pH}$ is $\sim 9$. As an alternative to the use a flocculant to achieve an ink with the suitable reological properties, we have dispersed the particles in a hydrogel (Pluronic). Pluronic is soluble in water at low temperatures and allows the dispersion of the ceramic particles when cold. At room temperature it forms a gel that can be used in the robocasting process. By controlling the amount of pluronic and the solid content it is possible to tailor the properties of the suspension for the printing process. The optimum solid content of the inks vary between $25-30 \%$ for the inks prepared using Pluronic and $~ 35$ vol\% for those prepared using PEI. In both cases, inks prepared with higher solid loading exhibited very limited printability and lower concentrations led to poor shape-retention.

Using these inks we have printed scaffolds with strut thickness ranging between 200 to $500 \mu \mathrm{m}$ and line spaces ranging between 75 to $500 \mu \mathrm{m}$ (Figure 3). Our preliminary tests indicate that the compressive strength of the robocasted grids ranges between 25 to $40 \mathrm{MPa}$. This is of the

order of porous calcium phosphates with similar porosity $(\sim 45 \%)$ fabricated by conventional 
techniques [2-10] and slightly higher than the corresponding value for cancellous bone (7-10 MPa) [20]. No de-bonding of the lines was observed during testing, indicating excellent adhesion between the printed lines. The structures prepared using Pluronic-based inks have usually larger volume of microporosiy in the printed line (Fig. 3). This is due to the larger organic content of the inks (up to $10 \mathrm{wt} \%$ ). The microporosity can affect negatively the compressive strength but several studies have shown that it can significatively enhance bone formation [21].

\section{Freeze Casting}

Freeze casting is a simple technique to produce porous complex-shaped ceramic or polymeric parts (Fig. 4). In freeze casting, a ceramic slurry is poured into a mold and then frozen. Under steady-state conditions, it is possible to grow ice crystals in the form of platelets, with a very high aspect ratio. The ice thus formed will have a lamellar microstructure, with the lamellae thickness depending mainly on the speed of the freezing front and the solid content of the ceramic suspension [15-17]. The frozen solvent acts temporarily as a binder to hold the part together for demolding. Subsequently, the part is subject to freeze-drying to sublimate the solvent under vacuum, avoiding the drying stresses and shrinkage that may lead to cracks and warping during normal drying. During freezing of the ceramic suspensions, the ceramic particles concentrate in the space between the ice crystals, creating a layered material (Fig. 4). After the ice is removed via sublimation and the green body is sintered, the result is a porous ceramic scaffold that exhibits striking similarities to the inorganic component of nacre's multilayered structure across a wide range of length scales (Fig. 5).

A critical step during processing is to achieve a stable suspension. If there is a significant amount of sedimentation during the process the solid content (and therefore the microstructure) will not be homogeneous along the freezing direction. The analysis of sedimentation of HA slurries (Fig. 5) has been used to identify optimum compositions. Low dispersant (0.5-1 wt\%) and high binder $(\sim 3-4 \mathrm{wt} \%)$ concentrations result in more stable dispersions suitable for the freeze casting process. However, if a very low dispersant concentration is used $(<0.5 \mathrm{wt} \%)$ it is difficult to achieve the high solid contents required to fabricate stable structures.

The pores are highly anisotropic and the pore channels can be characterized by two dimensional parameters (Fig. 5): the long axis (a) and the short axis (b). Depending on the cooling rates, the width of the long axis can typically vary between 100 to $600 \mu \mathrm{m}$ and the short axis between 5 to $50 \mu \mathrm{m}$. Like nacre, some bridges form between the layers, which are believed to increase fracture resistance. These characteristics contribute to the scaffold's mechanical strength, resulting in a material that can be up to four times stronger than the porous hydroxyapatite scaffolds with similar porosities currently used in bone substitutes (Fig. 7).

The final scaffold microstructure is a replica of the ice. Modifying the amount of water in the slurry as well as the shape of the solvent crystals will modify the final amount, shape and size of the porosity [15-17]. In particular, during the steady freezing regime, the ice crystals exhibit a homogeneous morphology throughout the whole sample resulting in a very homogenous lamellae thickness. This also means that the ratio between the thickness of the pore and the thickness of the ceramic lamellae is primarily determined by the initial slurry concentration. For instance, if the volume fraction of ice and ceramic particles is the same, layer thickness (e.g., 20 microns) will be the same as the porosity (20 microns in their smallest dimension). For low slurry 
concentration, the porosity becomes predominant and layer thickness decreases. The final porosity of the scaffolds ranges from 45 to 75 volume percent.

The microstructural features in freeze casting can be controlled by applying the physics of ice formation. In particular, the ice-tip radius (and as a consequence the thickness of the ice crystals), which is physically determined by the magnitude of supercooling ahead of the freezing front [22], can be modified by increasing or decreasing the cooling rate during freezing. For fast cooling rates, supercooling becomes larger and the width of the pores and the ceramic layers can be scaled down. On the other hand, under a very slow cooling regime the layer (or pore) thickness can be noticeably increased.

Klawitter and Hulbert [23] established a minimum pore size of $\sim 100 \mu \mathrm{m}$ for bone growth into ceramic structures and similar conclusion was reached by Simske et al. [24]. More recently, Itala claimed that bone ingrowth occurred in pores as small as $50 \mu \mathrm{m}$ [25] and similar results were obtained for porosity engineered in the range 15-40 $\mu \mathrm{m}$ [26]. The porosity must be interconnected to allow the ingrowth of cells, vascularization and diffusion of nutrients. Typical porosities for HA scaffolds described in the literature range from $35 \%$ [27, 28] to $75 \%$ [28] with pore sizes between $50 \mu \mathrm{m}$ [25] and $400 \mu \mathrm{m}$ [28]. A preponderance of pores this large can make the implant material rather weak. Moreover, even small movements of the implant (which are hard to avoid) can cause complications by cutting off blood supply to tissue in the pores, which can lead to inflammation. In addition, bone response reflects meso-scale structure and direct bone attachment and conduction along the struts of porous scaffolds has been reported [29], even for small porosity [26], revealing that pore connectivity and orientation are at least as important as pore size. Hence, the porosity range reported in this study might be suitable for osseous regeneration.

\section{Conclusions}

Porous hydroxyapatite scaffolds have been fabricated using two different techniques: robotic assisted deposition and freeze casting. While the porosity of the robocasted materials is designed using a computer, freeze casting allows the fabrication of strong lamellar porous materials with a structure that resembles the one of the inorganic component of nacre. Both techniques show promise for a large number of applications that require tailored scaffolds. Because the processes rely on physical rather than chemical interactions, they can be easily extended to any calcium phosphate or suitable ceramic for biomedical applications, opening the way to systematically manipulate the degradation rates and prepare materials with an optimum combination of mechanical properties and bioresorption. Control of the microporosity and roughness of the ceramic walls and struts will also help to manipulate cell attachment and osseointegration, issues that will be addressed in the future work.

\section{Acknowledgements}

This work was supported by the Director, Office of Science, Office of Basic Energy Sciences, of the U.S. Department of Energy under Contract No. DE-AC02-05CH11231 This work was supported by the National Institutes of Health (NIH) under Grant No. 5R01 DE015633. 


\section{References}

[1] Langer, R., and Vacanti, J. P., 1993, “Tissue Engineering,” Science, 260, pp. 920.

[2] Almirall, A., Larrecq, G., Delgado, J. A., Martinez, S., Planell, J. A., and Ginebra, M. P., 2004, "Fabrication of Low Temperature Macroporous Hydroxyapatite Scaffolds by Foaming and Hydrolysis of an alpha-TCP Paste," Biomaterials 25, pp. 3671-3680.

[3] del Real, R. P., Wolke, J. G., Vallet-Regi, M., and Jansen, J. A., 2002, "A New Method to Produce Macropores in Calcium Phosphate Cements," Biomaterials, 23, pp. 3673-3680.

[4] Ramay, H. R., and Zhang, M., 2003, "Preparation of Porous Hydroxyapatite Scaffolds by Combination of the Gel-Casting and Polymer Sponge Methods," Biomaterials, 24, pp. 32933302.

[5] Sous, M., Bareille, R., Rouais, F., Clement, D., Amedee, J., Dupuy, B., and Baquey, C., 1998, "Cellular Biocompatibility and Resistance to Compression of Macroporous $\square$-Tricalcium Phosphate Ceramics," Biomaterials, 19, pp. 2147-2153.

[6] Kawata, M., Uchida, H., Itatani, K., Okada, I., Koda, S., and Aizawa, M., 2004, "Development of Porous Ceramics with Well-Controlled Porosities and Pore Sizes from Apatite Fibers and their Evaluations," J. Mater. Sci. Mater. Med., 15, pp. 817-823.

[7] Le Huec, J. C., Schaeverbeke, T., Clement, D., Faber, J., and Le Rebeller, A., 1995, "Influence of Porosity on the Mechanical Resistance of Hydroxyapatite Ceramics Under Compressive Stress," Biomaterials, 16, pp. 113-120.

[8] Liu, D-M., 1997, "Influence of Porosity and Pore Size on the Compressive Strength of Porous Hydroxyapatite Ceramic," Ceramics International, 23, pp. 135.

[9] Milosevski, M., Bossert, J., Milosevski, D., and Gruevska, N., 1999, "Preparation and Properties of Dense and Porous Calcium Phosphate," Ceramics International, 25, pp. 693-696. [10] Bignon, A., Thesis, National Institute of Applied Science. Lyon, France, 2002.

[11] Michna, S.,Wu, W. and Lewis, J. A., 2005 "Concentrated hydroxyapatite inks for directwrite assembly of 3-D periodic scaffolds," Biomaterials, 26, pp. 5632-5639.

[12] Russias, J., Saiz, E., Deville, S., Gryn, K., Liu, G., Nalla, R. K., and Tomsia, A. P., 2007, "Fabrication and in vitro characterization of three-dimensional organic/inorganic scaffolds by robocasting," Journal of Biomedical Materials Research Part A, 83A, pp. 434-445.

[13] Smay, J. E., Cesarano, J., and Lewis, J. A., 2002, "Colloidal Inks for Directed Assembly of 3-D Periodic Structures," Langmuir, 18, pp. 5429.

[14] Miranda, P., Saiz, E., Gryn, K., and Tomsia, A. P., 2006, "Sintering and Robocasting of $\alpha-$ Tricalcium Phosphate Scaffolds for Orthopaedic Applications," Acta Biomaterialia, 2, pp. 457466.

[15] Deville, S., Saiz, E., Nalla, R. K., and Tomsia, A. P., 2006, "Freezing as a Path to Build Complex Composites," Science 311, pp. 515-518.

[16] Deville, S., Saiz, E., and Tomsia, A. P., 2007, "Ice-templated porous alumina structures," Acta Materialia, 55, pp.1965-1974.

[17] Deville, S., Saiz, E., and Tomsia, A. P., 2006, "Freeze casting of hydroxyapatite scaffolds for bone tissue engineering," Biomaterials, 27, pp. 5480-5489.

[18] H.I. Zhang HF, Brust M, Butler MF, Rannard SP, Cooper AI, Nat Mater 4(10) (2005), p.787-793

[19] Gutierrez, M.C., Jobbagy, M., Rapun, N., Ferrer, M. L. and del Monte, F., 2006, “A biocompatible bottom-up route for the preparation of hierarchical biohybrid materials,"

Advanced Materials, 18, pp. 1137-1140. 
[20] Murugan, R., and Ramakrishna, S., 2005, "Development of Nanocomposites for Bone Grafting," Composites Science and Technology, 65, pp. 2385-2390.

[21] Karageorgiou, V. and Kaplan, D."Porosity of 3D biomaterial scaffolds and osteogenesis", Biomaterials 26(27), September 2005, Pages 5474-5491

[22] Hunt, J. D., 1999, "Pattern Formation in Solidification,” Mater. Sci. Tech., 15, pp. 9-14.

[23] Klawitter, J. J., and Hulbert, S. F., 1971, "Application of Porous Ceramics for the Attachment of Load Bearing Internal Orthopedic Applications," J. Biomed. Mater. Res., 2, pp. 161-229.

[24] Simske, S. J., Ayers, R. A. and Bateman, T. A., 1997, "Porous Materials for Bone Engineering," in Porous Materials for Tissue Engineering, Dixit D-MLaV, editor. Enfield, NH: Transtech, pp. 151-182.

[25] Itala, A. I., Ylanen, H. O., Ekholm, C., Karlsson, K. H., and Aro, H. T., 2001, "Pore diameter of more than $100 \mu \mathrm{m}$ is not requisite for bone ingrowth in rabbits," J. Biomed. Mater. Res., 58, pp. 679-683.

[26] Tithi Dutta Roy., J. L. S., John, L., Ricci, E., Rekow, D., Van P. Thompson, and Parsons. R., 2003, "Performance of Degradable Composite Bone Repair Products Made Via ThreeDimensional Fabrication Techniques," J. Biomed. Mater. Res., 66A, pp. 283-291.

[27] Pilliar, R. M, Filiaggi, M. J., Wells, J. D., Grynpas, M. D. and Kandel, R. A., 2001, "Porous Calcium Polyphosphate Scaffolds for Bone Substitute Applications - In Vitro Characterization," Biomaterials, 22, pp. 963-972.

[28] Tamai N, Myoui, A., Tomita, T., Nakase, T., Tanaka, J., Ochi, T. and Yoshikawa, H., 2002, "Novel Hydroxyapatite Ceramics with an Interconnective Porous Structure Exhibit Superior Osteoconduction In Vivo,” J. Biomed. Mater. Res., 59, pp. 110-117.

[29] Zmora, S., Glicklis, R., and Cohen, S., 2002, "Tailoring the Pore Architecture in 3-D Alginate Scaffolds by Controlling the Freezing Regime During Fabrication,” Biomaterials, 23, pp. 4087-4094. 


\section{Figure Captions}

Figure 1. Schematic of the robocasting process. A ceramic slurry is extruded through a thin nozzle to build a part layer-by-layer following a computer design.

Figure 2. Hydroxyapatite parts with complex geometries can be printed by robocasting. The picture shows a part with a gradient in porosity after printing and sintering.

Figure 3. Scanning electron micrographs of (a) the cross section and (b) the surface of a robocasted hydroxyapatite scaffold processed with PEI-Methocell. Comparison between the microstructures of specimens printed with (c) PEI-Methocell and (d) with Pluronic gel.

Figure 4. (a) Schematic of the experimental apparatus employed to directionally freeze ceramic slurries while controlling the speed of the freezing front. The ceramic slurry is poured into a Teflon mold placed between two copper cold fingers whose temperature is controlled by liquid nitrogen baths and ring heaters. (b) Schematic of the process. While the ceramic slurry is freezing, the growing ice crystals expel the ceramic particles, creating a lamellar microstructure oriented in a direction parallel to the movement of the freezing front. For a highly concentrated slurries, the interaction between particles becomes critical: A small fraction of particles are entrapped within the ice crystals by tip-splitting and subsequent healing, leading to the formation of inorganic bridges between adjacent walls.

Figure 5. Microstructure of a porous hydroxyapatite scaffold fabricated by freeze casting.

Figure 6. Equilibrium sediment height (after $10 \mathrm{~h}$ ) of $40 \mathrm{wt} \%$ HA slurries as a function of the dispersant and binder concentration. The darker blue areas identify the regions with higher sediment (more stable slurries).

Figure 7. Compressive strength of lamellar HA scaffolds prepared by freeze casting vs. reported values for materials fabricated using conventional techniques [2-10]. The typical pore sizes of conventional porous HA scaffolds are on the order of 100 to $800 \mu \mathrm{m}$ in order to allow bone ingrowth. For the lamellar materials, compression is applied in the direction parallel to the ceramic layers. The presence of inorganic bridges between the ceramic layers prevents Euler buckling of the ceramic layers and contributes to the high strength. 


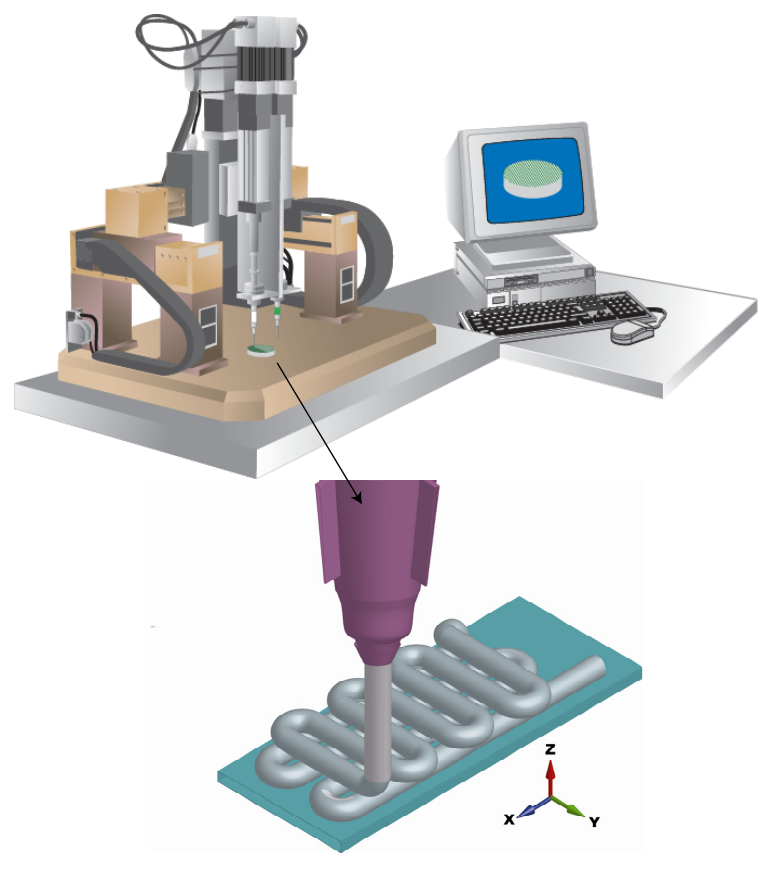

Figure 1 


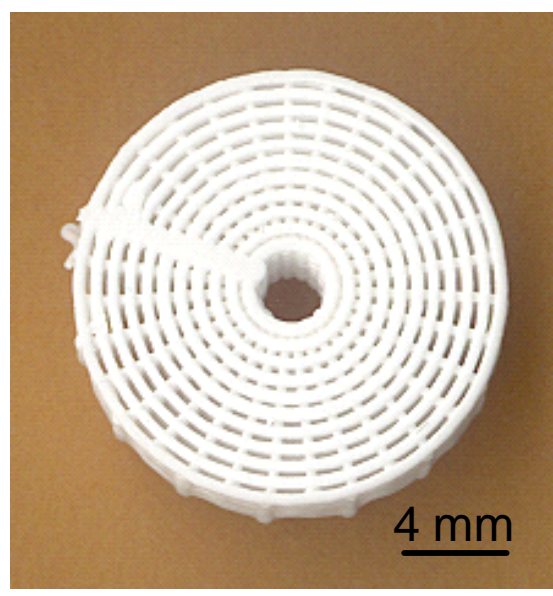

Figure 2 


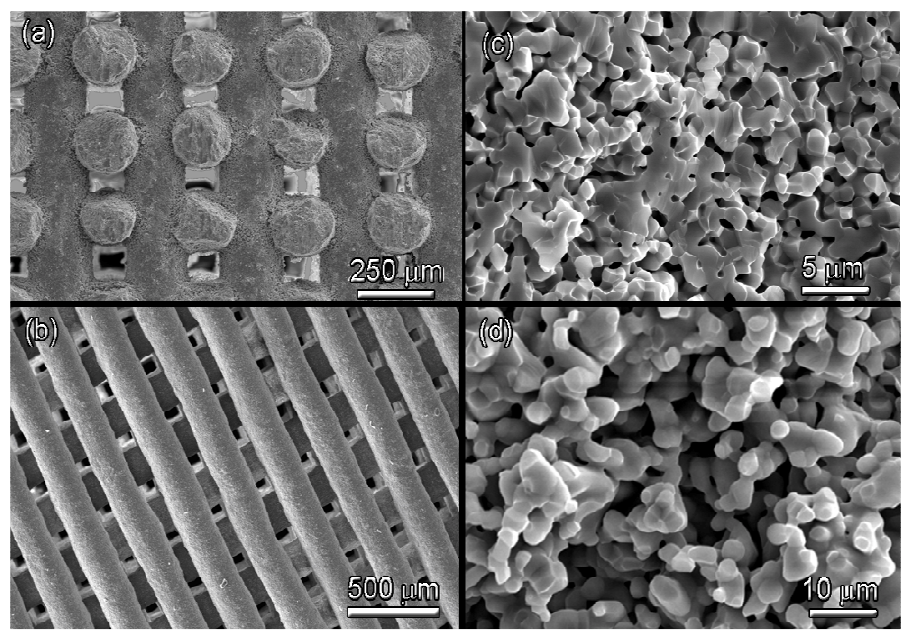

Figure 3 
(a)

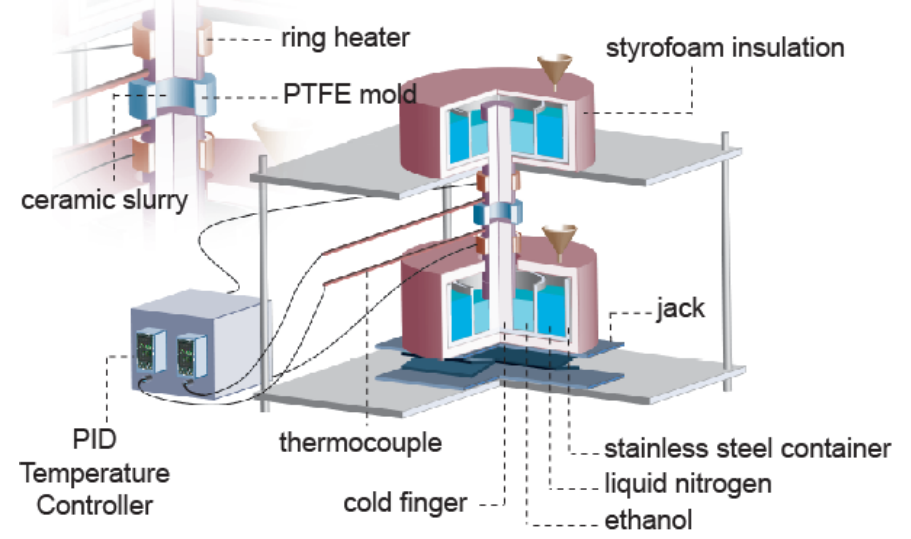

(b)

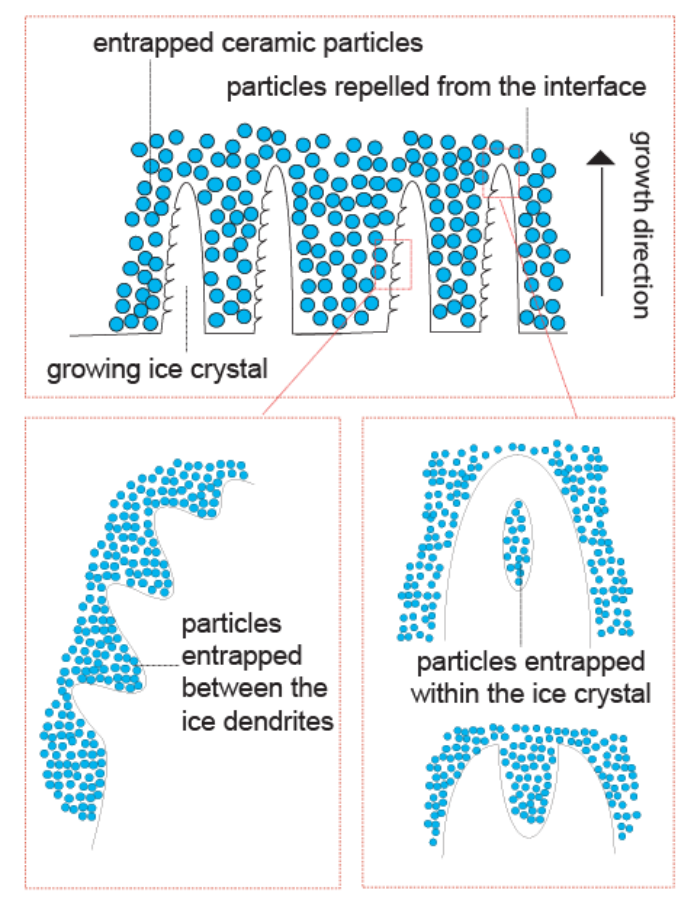

Figure 4 


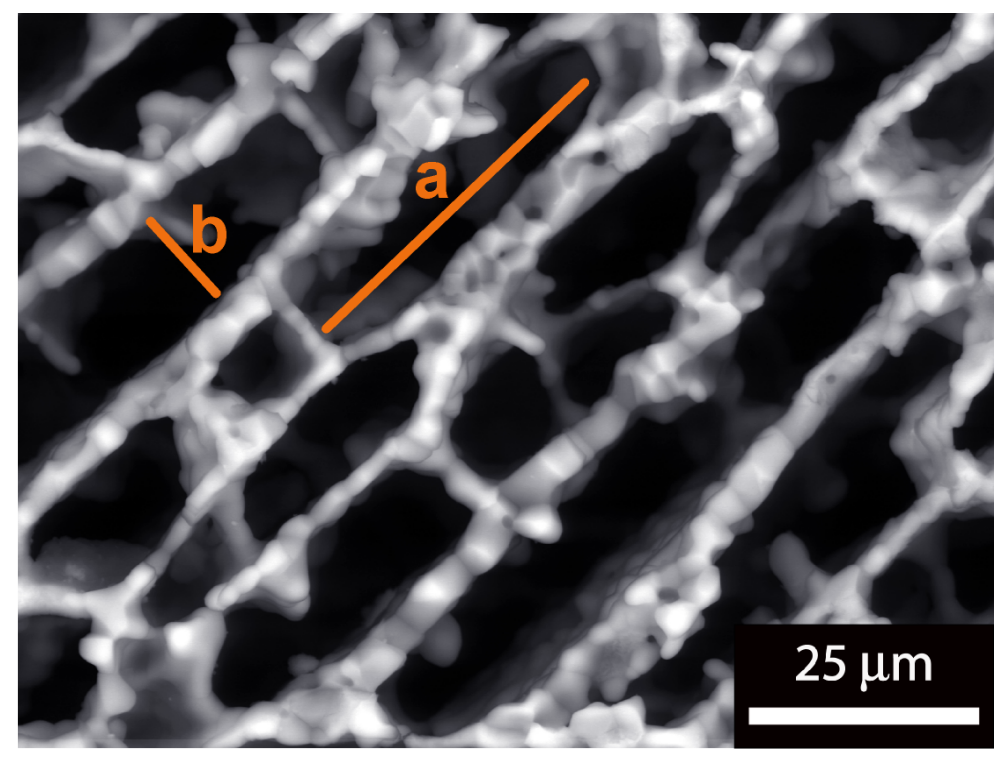

Figure 5 


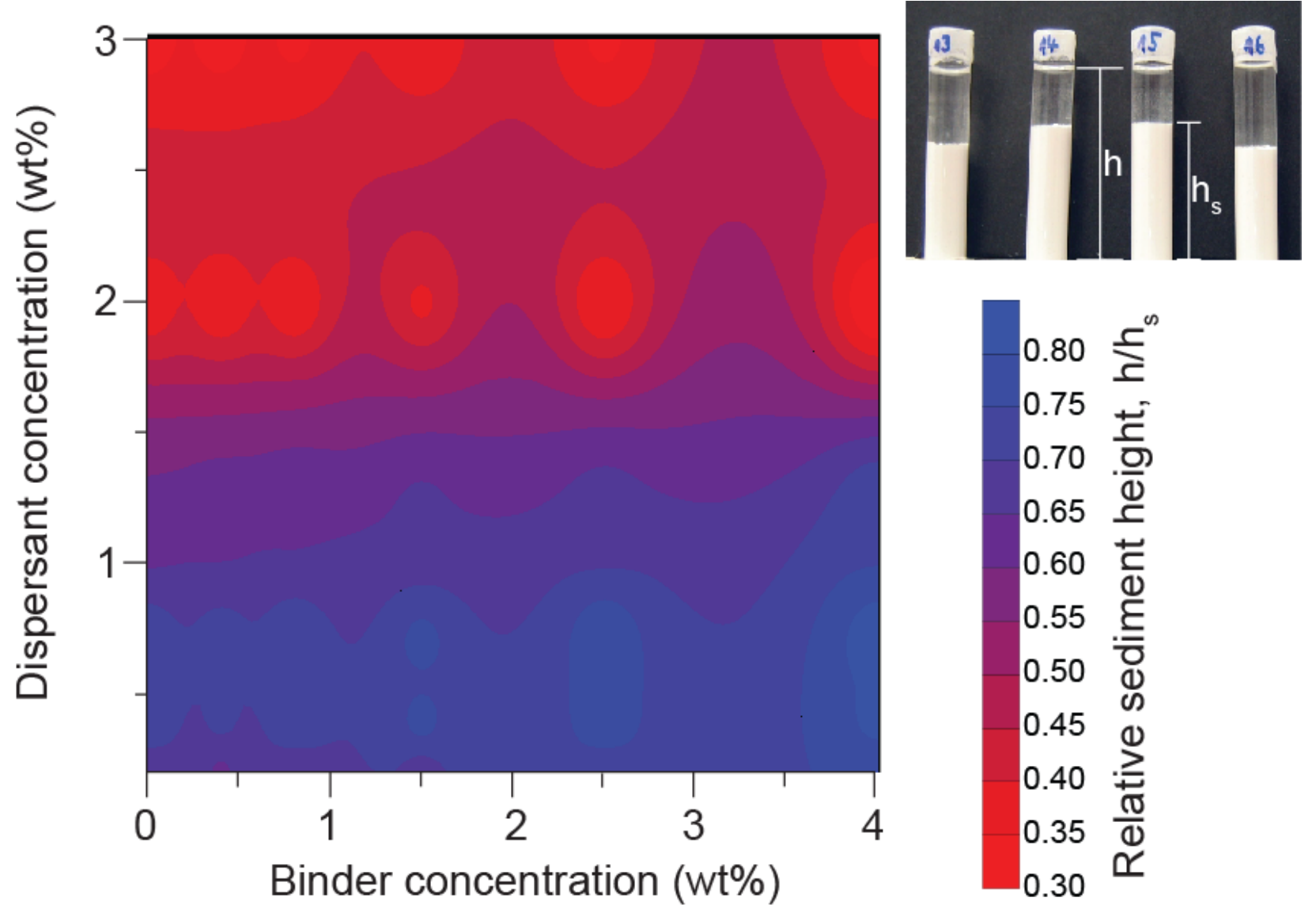

Figure 6 


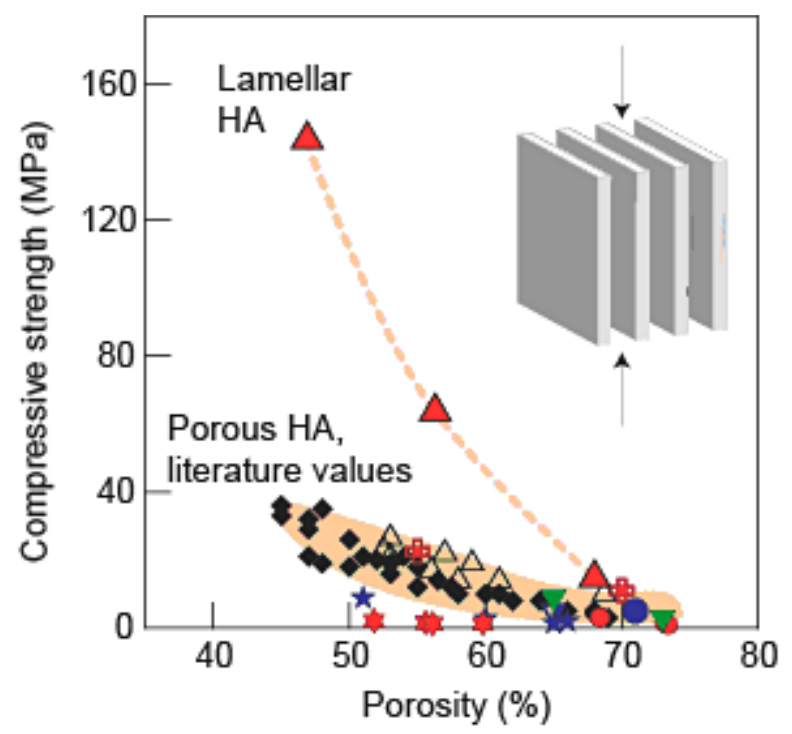

Figure 7 